Research Paper

\title{
Clavulanic acid production by the MMS 150 mutant obtained from wild type Streptomyces clavuligerus ATCC 27064
}

\author{
Eliton da Silva Vasconcelos ${ }^{1}$, Vanderlei Aparecido de Lima ${ }^{1}$, Leandro Seiji Goto ${ }^{1,2}$, \\ Isara Lourdes Cruz-Hernández ${ }^{1}$, Carlos Osamu Hokka ${ }^{1}$ \\ ${ }^{1}$ Departamento de Engenharia Química, Universidade Federal de São Carlos, São Carlos SP, Brazil. \\ ${ }^{2}$ Grupo de Biofísica Molecular "Sérgio Mascarenhas", Instituto de Física de São Carlos, \\ Universidade de São Paulo, São Carlos, SP, Brazil. \\ Submitted: March 09, 2011; Approved: April 01, 2013.
}

\begin{abstract}
Clavulanic acid (CA) is a powerful inhibitor of the beta-lactamases, enzymes produced by bacteria resistants to penicillin and cefalosporin. This molecule is produced industrially by strains of Streptomyces clavuligerus in complex media which carbon and nitrogen resources are supplied by inexpensive compounds still providing high productivity. The genetic production improvement using physical and chemical mutagenic agents is an important strategy in programs of industrial production development of bioactive metabolites. However, parental strains are susceptible to loss of their original productivity due genetic instability phenomenona. In this work, some $S$. clavuligerus mutant strains obtained by treatment with UV light and with MMS are compared with the wild type (Streptomyces clavuligerus ATCC 27064). The results indicated that the random mutations originated some strains with different phenotypes, most divergent demonstrated by the mutants strains named AC116, MMS 150 and MMS 54, that exhibited lack of pigmentation in their mature spores. Also, the strain MMS 150 presented a larger production of CA when cultivated in semi-synthetics media. Using other media, the wild type strain obtained a larger CA production. Besides, using the modifed complex media the MMS 150 strain showed changes in its lipolitic activity and a larger production of CA. The studies also allowed finding the best conditions for a lipase activity exhibited by wild type $S$. clavuligerus and the MMS150 mutant.
\end{abstract}

Key words: clavulanic acid, Streptomyces clavuligerus, mutation, lipase.

\section{Introduction}

From all the antibiotics actually being extracted from microorganisms, about $66 \%$ come from actinomycetes, $22 \%$ from fungi and $12 \%$ from non-actinomycetales bacteria. About $80 \%$ of the antibiotics produced from actinomycetes come from the Streptomyces genus (Kieser et al., 2000). Streptomyces also represents the greatest actinomycetes group which produces several other classes of biologically active secondary metabolites (Williams et al., 1989, Korn-Wendisch and Kutzner, 1992).

Since the beginning of the first clinical applications, the efficiency of the $\beta$-lactam antibiotics has been declining, due to the astonishing increasing number of bacteria capable of $\beta$-lactam resistance. Such resistance is due to pathogenic microorganisms secreting of $\beta$-lactamase enzymes, the most important mechanism of bacterial $\beta$-lactam resistance (Brock et al., 1994).

Thus, there is an important number of pathogens resistant to $\beta$-lactam antibiotics, including Staphylococcus aureus, Escherichia coli, Klebsiella pneumoniae, Proteus vulgaris, Proteus mirabilis and Haemophilus influenzae. All of these microorganisms produce $\beta$-lactamases that hydrolyze the $\beta$-lactamic ring from antibiotics, releasing compounds that lack the original antibiotic activity (Bush, 1989).

The initial (and not successful) attempts to find a $\beta$-lactamase inhibitor date from 1940. In 1967 began the first program of microorganism screening trials for the iso- 
lation of naturally occurring $\beta$-lactamase inhibitor producers. This research resulted in the isolation of the substances clavulanic acid from Streptomyces clavuligerus and olivanic acid from Streptomyces olivaceus (Rolinson, 1991), the latter never used clinically due to rapid renal degradation rates.

Despite of being a very weak antibiotic (Minimal Inhibitory Concentration around $25-125 \mu \mathrm{g} / \mathrm{mL}$ ), clavulanic acid is a powerful $\beta$-lactamase inhibitor acting on penicillin and cephalosporin resistant bacteria, being frequently used in combination with these. Clavulanic acid is a suicide inhibitor that binds irreversibly to a hydroxyl group from a specific serine residue within the catalytic core of serine$\beta$-lactamases, producing a stable inactive enzyme (Foulstone and Reading, 1982; Baggaley et al., 1997).

Clavulanic acid shows a great affinity to proteins that bind to penicillin derivatives, favoring its use in combination with semi-synthetic penicillins. The most known current combination in clinical use is in combination with amoxicillin (Elander, 2003).

Lipids and oils are now considered essential media components in the antibiotic industry because they possess natural antifoam properties, are a cheaper alternative carbon source when compared with carbohydrates and may increase secondary metabolite titres (Pan et al., 1959).

Microbial lipases recently have attracted considerable attention owing to their biotechnological potential, ranging from the use in laundry detergents to stereospecific biocatalysts (Jaeger et al., 1994). These lipases are secreted into the culture medium by many fungi and bacteria (Jaeger et al., 1996). They differ from one to another in their physical and biochemical properties (Aires-Barros et al., 1994). As each industrial application requires specific properties of lipases, there is still an interest in additional lipases that could be used in new applications.

In the clavulanic acid biosynthesis, glycerol is regarded as a rate limiting substrate and a component of primary importance in this process (Maranesi et al., 2005). Long chain triglycerides at lipid/water interfaces are hydrolyzed by lipases (EC 3.1.1.3), producing fat acids and glycerol. These enzymes that catalyze the hydrolysis of vegetable oils are also produced by Streptomyces species (Large et al., 1999).

This work presents an investigation on the clavulanic acid production of some Streptomyces clavuligerus mutant strains obtained by treatment with UV and MMS and the comparison of these with the productivity of the wild type strain. Additionally, the lipase activity of both was studied and modeled.

\section{Material and Methods}

\section{Microorganism strains}

Streptomyces clavuligerus ATCC 27064 (www.atcc.org) was used as standard strain in all the exper- iments. Mutant strains were supplied by Dr. Isara Lourdes Cruz-Hernández, Universidade Estadual de São Paulo (UNESP), Depto. de Tecnologia Química, IQ - Araraquara, Araraquara, SP, Brazil. The mutant strains present here were as follows: AC 116, MMS 150 e MMS 54. AC116 was obtained by UV light mediated mutation described by Lee et al. (2002). MMS 150 and MMS 54 strains were obtained by chemical mutagenesis using Methyl methanesulphonate following the technique described by Stonesifer \& Baltz (1985). All of the mutant strains when cultivated in solid medium, lacked pigmentation in their mature spores (data not shown), being classified as whi mutants ("white" Chater, 2001). The wild type strain is classified in the gray series of category IV from Streptomyces genera, based on the dark greenish pigmentation shown by its mature spores (Locci, 1989). All of the microorganisms were conserved as vegetative cell suspensions in cryoprotective $10 \% \mathrm{p} / \mathrm{v}$ glycerol stocks stored at $-70^{\circ} \mathrm{C}$.

\section{Culture media}

All the microorganisms were initially cultivated in reactivation medium (Rosa et al., 2005, composition in g. $\mathrm{L}^{-1}$ in distilled water), glycerol, 15.0; bacto-peptone, 10.0; malt extract 10.0; yeast extract 1.0; $\mathrm{K}_{2} \mathrm{HPO}_{4}, 2.5 ; \mathrm{MgSO}_{4} .7 \mathrm{H}_{2} \mathrm{O}$, 0.75; $\quad \mathrm{MnCl}_{2} .4 \mathrm{H}_{2} \mathrm{O}, \quad 0.001 ; \quad \mathrm{FeSO}_{4} .7 \mathrm{H}_{2} \mathrm{O}, \quad 0.001$; $\mathrm{ZnSO}_{4} .7 \mathrm{H}_{2} \mathrm{O}, 0.001$; in MOPS buffer, 21.0 (100 mM). Medium $\mathrm{pH}$ was adjusted to $\mathrm{pH} 6.8$ with titration of a $\mathrm{NaOH}$ $5 \mathrm{M}$ solution and sterilized. Reactivated cells were then inoculated in the respective mediums used in the clavulanic acid production assays.

In the first phase of this work was used the complex culture medium proposed by Maranesi et al. 2005 (medium composition in g.L $\mathrm{L}^{-1}$ in distilled water): soy flour, 20.0; glycerol, 10.0; $\mathrm{K}_{2} \mathrm{HPO}_{4}$, 1.2; soy oil, 23.0; $\mathrm{MnCl}_{2} .4 \mathrm{H}_{2} \mathrm{O}$, $0.001 ; \mathrm{FeSO}_{4} .7 \mathrm{H}_{2} \mathrm{O}, 0.001 ; \mathrm{ZnSO}_{4} .7 \mathrm{H}_{2} \mathrm{O}, 0.001,21.0$ (100 mM) MOPS buffer, pH 6.8.

In the second phase, three different semy-sinthetic mediums were used in the clavulanic acid production assays, GSPG, GSPO and GSPA (Romero et al., 1986). GSPG medium composition was (g. $\mathrm{L}^{-1}$ in distilled water): glycerol, 15.0; sucrose, 20.0; proline, 2.5; glutamic acid, 1.5; NaCL, 5.0; $\mathrm{K}_{2} \mathrm{HPO}_{4}, 2.0 ; \mathrm{CaCl}_{2}, 0.4 ; \mathrm{MnCl}_{2} .4 \mathrm{H}_{2} \mathrm{O}$, 1.0; $\mathrm{FeCl}_{3} .6 \mathrm{H}_{2} \mathrm{O}, 0.1 ; \mathrm{ZnCl}_{2}, 0.05 ; \mathrm{MgSO}_{4} .7 \mathrm{H}_{2} \mathrm{O}, 1.0 ; \mathrm{pH}$ 7.0. GSPO and GSPA mediums compositions were the same as GSPG, except of containing $10 \mathrm{mM}$ ornitine or arginine respectively, in place of glutamic acid from original GSPG.

\section{Culture conditions}

Batch cultivations for the $S$. clavuligerus clavulanic acid production were done in a table top rotatory shaker G-25 (New Brunswich Scientific Co). Assays were done in three steps, strain reactivation, microorganism growth and clavulanic acid production. 
In the reactivation step, $3.5 \mathrm{~mL}$ of the $S$. clavuligerus vegetative cell suspension stock were inoculated in $500 \mathrm{~mL}$ Erlenmeyers containing $50 \mathrm{~mL}$ of reactivation medium and incubated for $24 \mathrm{~h}$ at $28^{\circ} \mathrm{C}, 250 \mathrm{rpm}$.

In the growth step, $5 \mathrm{~mL}$ of the reactivatated $S$. clavuligerus suspensions were separately transferred to $500 \mathrm{~mL}$ Erlenmeyers containing $45 \mathrm{~mL}$ of culture mediums, each with the same composition relative to main clavulanic acid production mediums. The growth step was carried over $24 \mathrm{~h}$ at $28^{\circ} \mathrm{C}, 250 \mathrm{rpm}$.

Finally, in the production step, the hole growth culture volumes were transferred into Erlenmeyers containing the production mediums, beginning the main clavulanic acid production process. Clavulanic acid production cultures were carried over $120 \mathrm{~h}$ and samples from the cultures were collected every $12 \mathrm{~h}$.

\section{Genetic markers amplification}

Streptomyces clavuligerus ATCC 27064 was purchased from the American Type Culture Collection (Manassas, VA) and stored at $-80^{\circ} \mathrm{C}$ in $40 \% \mathrm{v} / \mathrm{v}$ glycerol. $S$. clavuligerus MMS 150 mutant was supplied by Dr. Isara Lourdes Cruz-Hernández and is an ATCC 27064 derivative mutant strain obtained from MMS (methyl methanesul- fonate) treatment. Custom oligonucleotides were provided by IDT (Integrated DNA Technologies - Coralville, IA). PCR reagents were supplied by Fermentas (Glen Burnie, MD). DMSO, was purchased from Sigma (St. Louis, MO). Other reagents were of analytical grade or higher. Standard recombinant DNA techniques used throughout this work were previously described (Ausubel, 2002), or are detailed in the text. The genetic markers used here for PCR diagnosis have the following GenBank sequence access codes: AY426768 (pah1, 1056 bp), X84101.1 (cas2, 978 bp), AY034175.1 (orf10, 1224 bp and orf11, 207 bp; 5 bp be-

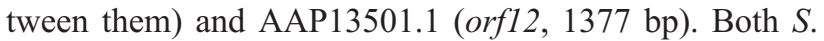
clavuligerus ATCC 27064 and S. clavuligerus MMS 150 were cultivated for $72 \mathrm{~h}$ at $28^{\circ} \mathrm{C}$ in ISP2 medium for the isolation of genomic DNA. The cells were collected via centrifugation and washed with phosphate-buffered saline $(50 \mathrm{mM}$ sodium phosphate, $150 \mathrm{mM} \mathrm{NaCl})$. Approximately $200 \mathrm{mg}$ cells (wet weight) were used for DNA extraction, using the Wizard Genomic DNA extraction kit (Promega). The oligonucleotides were all designed to incorporate an NdeI restriction site at the 5' and of the PCR product and an XhoI site at the 3' end, right after the predicted stop codons for further cloning experiments not related here. The oligonucleotides used were as follows:

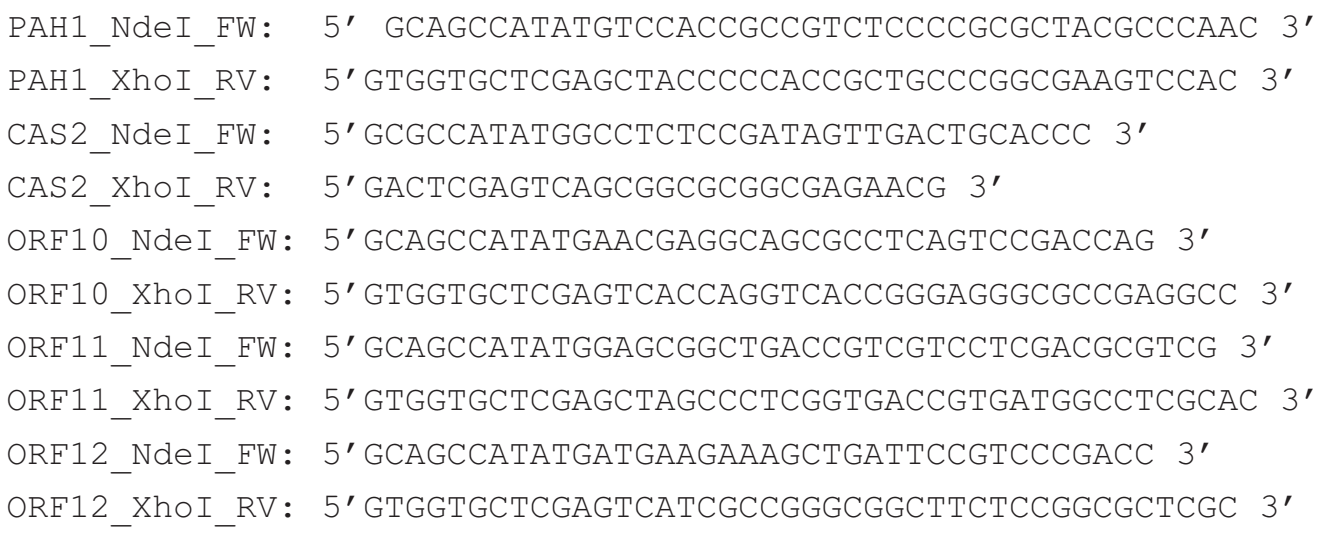

Each of the amplifications was done with oligonucleotides used in pairs named according to the targeted gene, except for ORF10-11 amplification in which ORF10_NdeI_FW was used with ORF11_XhoI_RV to amplify both ORF10 and ORF11 fused by a 5 bp spacing region (Li et al., 2000). PCR was performed using a Mastercycler thermocycler (Eppendorf - Hamburg, Germany), programmed to execute a $98^{\circ} \mathrm{C}$ initial 5 min denaturing step, followed by 40 cycles of $94^{\circ} \mathrm{C}$ for $30 \mathrm{~s}, 65^{\circ} \mathrm{C}$ for $1 \mathrm{~min}, 72^{\circ} \mathrm{C}$ for $3 \mathrm{~min}$, and a final elongation step of $10 \mathrm{~min}$ at $72^{\circ} \mathrm{C}$. All the amplifications were done using $500 \mathrm{ng}$ of either $S$. clavuligerus ATCC 27064 or S. clavuligerus MMS150 genomic DNA and $50 \mathrm{pMol}$ of each primer in $20 \mu \mathrm{L}$ individual reactions containing reagents recommended by the supplier (Taq-HiFi, Fermentas), except for DMSO which was added up to $5 \% \mathrm{v} / \mathrm{v} .2 \mu \mathrm{L}$ of each amplification reaction were subjected to TAE $0.8 \%$ agarose electrophoresis.

\section{Biomass, clavulanic acid and lipase determination}

Cellular biomass was analyzed the dry weight method, collecting cells by centrifugation of the samples and washing the cells twice with double distilled water. The samples were then incubated at $65{ }^{\circ} \mathrm{C}$ until reaching the measured constant weight.

Glycerol concentrations were determined by HPLC, using a Shodex KS-802 (Lonpak) column monitoring the elution with a W410 refractometer detector. the column was pre equilibrated with $\mathrm{NaOH} 1 \mathrm{mM}$, eluent flow rate was $1 \mathrm{~mL} \mathrm{~min}{ }^{-1}$. The equipment was set to $80^{\circ} \mathrm{C}$. A reference calibration curve was obtained using analytical grade glycerol dilutions in destiled water ranging from 0.1 to 1.0 g. $\mathrm{L}^{-1}$ in the same conditions.

Clavulanic acid concentrations were determinated by HPLC using the Foulstone and Reading (1982) method, in a C-18 $\mu$-Bondapack column. Mobile phase was a metha- 
nol/phosphate buffer mix, at $2.5 \mathrm{~mL} / \mathrm{min}^{-1}$ flow rate. Elution temperature was kept at $28{ }^{\circ} \mathrm{C}$ monitoring at $311 \mathrm{~nm}$ wavelenght. A reference calibration curve was previously done using an amoxicillin/potassium clavulanate mix (the content of Clavulin $250 \mathrm{mg}$ - Smith Kline-Beecham do Brasil Ltda).

Extracellular lipase enzymatic activity was inferred by spectrophotometry using $p$-nitrophenil palmitate (pNPP) substrate monitoring the samples absorbance at $410 \mathrm{~nm}$ at $37^{\circ} \mathrm{C}$, as described by Gupta et al. (2002).

\section{Statistical analysis}

Statistical analyses were done using the XLSTAT v5.2 software. Student's $t$-distribution and Fisher tests were used in the lipase activity results comparisons.

\section{Experimental design and optimization}

$\mathrm{pH}$ and temperature, were the factors used as variables independents to optimize the extracellular lipase activity by Streptomyces clavuligerus. A $2^{2}$ full factorial central composite rotary design (CCRD) for two independent variables, each at five levels, was employed to fit a second order polynomial model which indicated that 11 experiments were required for this procedure (Table 1). The temperature and $\mathrm{pH}$ are factors that affect the lipase activity (Mingrui Yu et al., 2007).

The optimum $\mathrm{pH}$ e temperature for lipase activity by Streptomyces clavuligerus were determined by means of CCRD and RSM (response surface methodology). RSM consists of a group of mathematical and statistical techniques that can be used to define the relationships between the response and the independent variables. RSM defines the effect of the independent variables, alone or in combination, on the processes. In addition to analyzing the effects of the independent variables, this experimental methodology also generates a mathematical model. The graphical perspective of the mathematical model has led to the term Response Surface Methodology (Bas and Boyaci, 2007). A second-order polynomial model obtained through a multiple regression technique using the STATISTICA 7.0 software was used to describe the response surface. For two factors, the model obtained is expressed as follows:

$Y=b_{0}+b_{1} x_{1}+b_{2} x_{2}+b_{11} x_{1}^{2}+b_{22} x_{2}^{2}+b_{12} x_{1} x_{2}$

where $Y$ is the predicted response, $b_{0}, b_{1}, b_{2}, b_{11}, b_{22}, b_{12}$ are constant coefficients, and $x_{1}, x_{2}$ are the coded independent variables or factors.

The test factors were coded according to the following regression equation:

$$
x_{i}=\left(\frac{X_{i}-X_{0}}{\Delta X_{i}}\right)
$$

where $x_{i}$ is the coded value and $X_{i}$ is the actual value of the $i$ th independent variable, $X_{0}$ is the actual value at the center point, and $\Delta X_{i}$ is the step change value.

The fit quality of the second-order model equation was expressed by $\mathrm{R}^{2}$, the coefficient of determination, and its statistical significance was determined by an $F$-test. The significance of the regression coefficients were tested by analysis of variance (ANOVA) and $t$-test.

\section{Results}

\section{DNA analysis}

PCR amplification based on five clavulanic acid related genes of Streptomyces clavuligerus was done for validation of MMS150 mutant as a S. clavuligerus derivative strain. The targeted genes chosen were: pah1, cas2, orf10, orf10-11, orf11 and orf12. Information about these genes were previously published (Tahlan et al., 2004; Paradkar, 1995; Li, 2000).

Table 1 - Process variables used in the CCD, showing the treatment combinations and the mean experimental responses.

\begin{tabular}{|c|c|c|c|c|c|c|}
\hline \multirow[t]{2}{*}{ Treatment } & \multicolumn{2}{|c|}{ Coded setting levels $\mathrm{x} 1=\mathrm{T} ; \mathrm{x} 2=\mathrm{pH}$} & \multicolumn{2}{|c|}{ Actual levels $\mathrm{X} 1=\mathrm{T}\left({ }^{\circ} \mathrm{C}\right) ; \mathrm{X} 2=\mathrm{pH}$} & \multirow{2}{*}{$\begin{array}{l}\text { Extracellular lipase activ- } \\
\text { ity by } S \text {. clavuligerus wild } \\
\text { type }(\mathrm{U} / \mathrm{mL} \mathrm{h})\end{array}$} & \multirow{2}{*}{$\begin{array}{c}\text { Extracellular lipase activ- } \\
\text { ity by } S . \text { clavuligerus mu- } \\
\text { tant MMS } 150(\mathrm{U} / \mathrm{mL} \mathrm{h})\end{array}$} \\
\hline & $\mathrm{x} 1$ & $\mathrm{x} 2$ & $\mathrm{X} 1$ & $\mathrm{X} 2$ & & \\
\hline 1 & -1 & 1 & 8.5 & 27.9 & 2040.15 & 2140.91 \\
\hline 2 & -1 & -1 & 6.0 & 27.9 & 738.64 & 1745.45 \\
\hline 3 & 1 & 1 & 8.5 & 42.1 & 2068.94 & 1770.45 \\
\hline 4 & 1 & -1 & 6.0 & 42.1 & 981.06 & 656.82 \\
\hline 5 & 0 & 0 & 7.2 & 35.0 & 2096.97 & 1652.65 \\
\hline 6 & 0 & 0 & 7.2 & 35.0 & 2242.42 & 1621.97 \\
\hline 7 & 0 & 0 & 7.2 & 35.0 & 2140.15 & 1683.33 \\
\hline 8 & 0 & -1.41 & 5.5 & 35.0 & 1146.21 & 631.82 \\
\hline 9 & 0 & 1.41 & 9.0 & 35.0 & 2223.48 & 2348.48 \\
\hline 10 & -1.41 & 0 & 7.2 & 25.0 & 2498.48 & 1984.09 \\
\hline 11 & 1.41 & 0 & 7.2 & 45.0 & 2139.39 & 1517.42 \\
\hline
\end{tabular}


The PCR product visualization of these is shown in Figure 1. The results showed the same amplification profile for the two strains, S. clavuligerus wild type and S. clavuligerus MMS 150. Each PCR product showed a main amplification band of the expected mass under electrophoresis.

\section{Production of clavulanic acid}

All of the strains (mutants and the wild type) were firstly cultivated in a complex medium for comparison of their clavulanic acid production (Figure 2). The relative production of the MMS 150 mutant was the greatest among all the assayed mutants so that it was chosen for the further experiments in this work.

There was a great induction of lipase production, probably due to the presence of soybean oil in the culture medium (Large et al., 1999). However, the mutant strain had shown a drop on the clavulanic acid production around $36 \mathrm{~h}$ of cultivation. Glycerol consumption was around the same for both, the wild type strain showed a greater growth (Figure 3).

The wild type strain showed a simultaneous drop in the production of both clavulanic acid and lipase coincident with the depletion of glycerol. So, another cultivation study in complex medium deprived of glycerol was done. The results for the mutant strain show a greater lipase production when compared with the unmodified medium with a

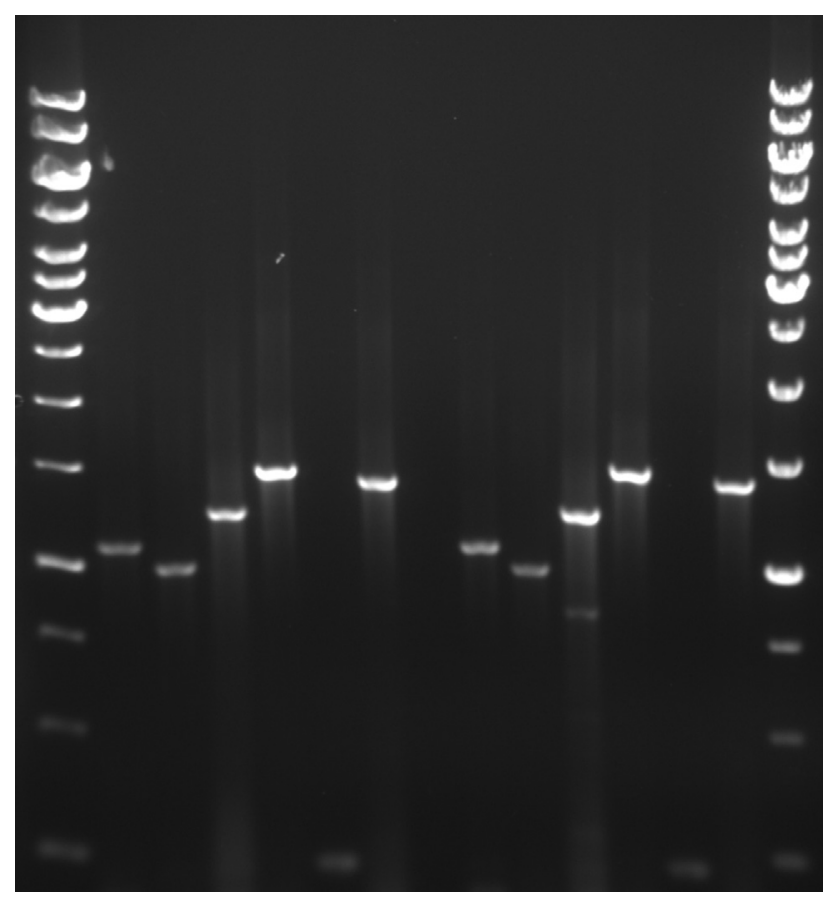

Figure 1 - Clavulanic acid related genetic markers PCR amplification products electrophoresis. $2 \mu \mathrm{L}$ samples from each PCR product were submitted to 0.8 agarose electrophoresis. Lanes M - Molecular mass markers (Generuler $1 \mathrm{~kb}$ - Fermentas). Lanes 1-6 are respective to amplification of pah1, cas2, orf10, orf10-11, orf11 and orf12 genetic markers using Streptomyces clavuligerus ATCC 27064 genomic DNA. Lanes 7-12 are respective to the amplification of the same genetic markers using genomic DNA from $S$. clavuligerus MMS 150. greater clavulanic acid production, leading to a suggestive correlation that lipase activity levels were elevated in the presence of oil as main source of carbon (Large et al., 1999). On the other hand, the wild type strain showed smaller values of biomass, lipolytic activity and clavulanic acid production when cultivated in the same complex medium lacking glycerol (Figure 4).

The clavulanic acid production in the GSPA, GSPG and GSPO semi-synthetic mediums was all time smaller when compared to the complex medium. However, in all of them the mutant strain has shown greater production, especially when cultivated in GSPA medium, where the clavulanic acid production was about six fold higher than the wild type strain after $60 \mathrm{~h}$ fermentation. Glycerol consumption and biomass data are also shown in Figure 5.

\section{Lipase activity optimization on S. clavuligerus}

Based on the previous studies, temperature and $\mathrm{pH}$ were identified as the main factors affecting lipase activity

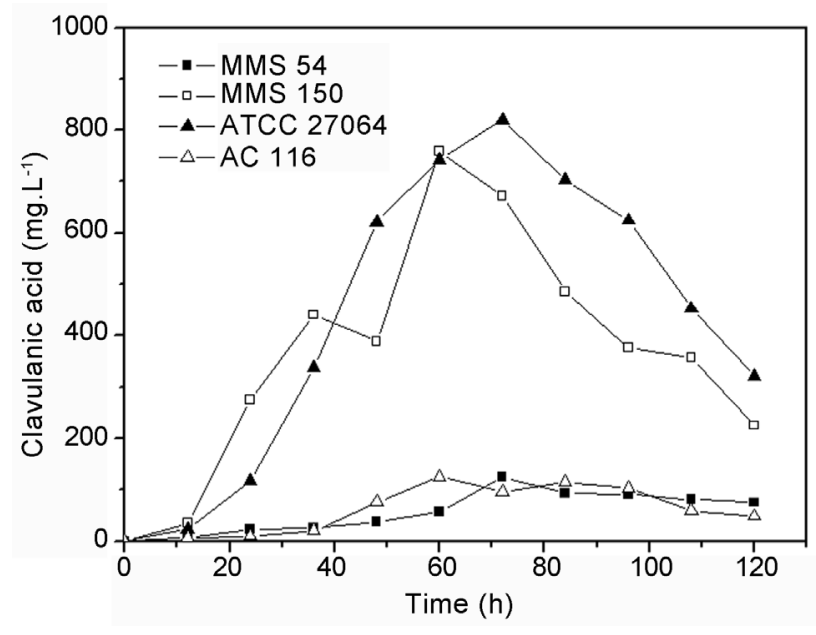

Figure 2 - Comparison of clavulanic acid concentration amongst all the $S$. clavuligerus strains.

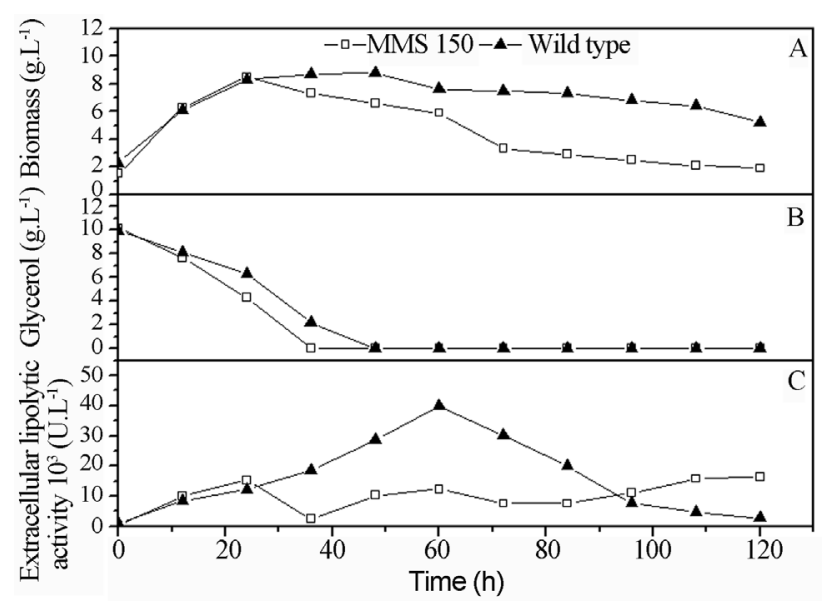

Figure 3 - Comparison of the biomass (a), glycerol consumption (b) and lipolytic activity (c) between the mutant and wild type strains cultivated in complex medium. 


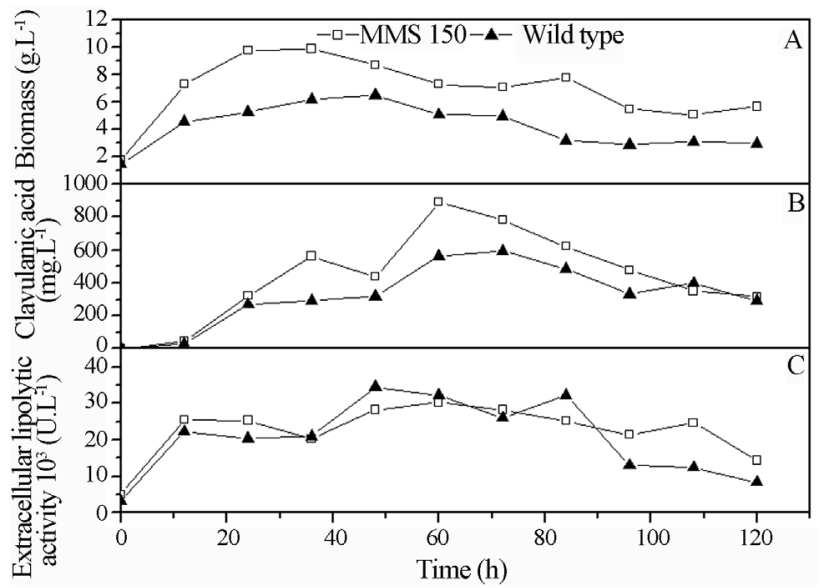

Figure 4 - Comparison of the biomass (a), clavulanic acid (b) and lipolytic activity (c) between the mutant and wild type strains cultivated in the modified complex medium lacking glycerol.

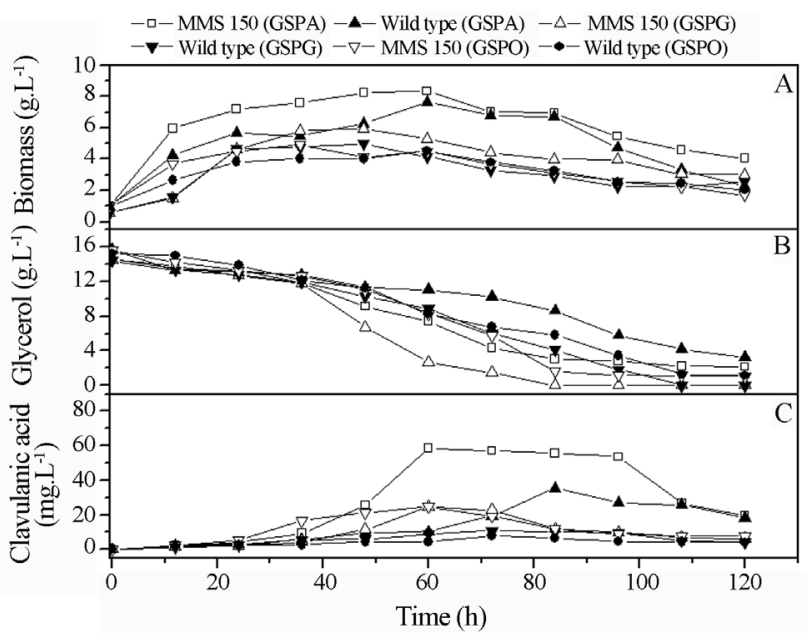

Figure 5 - Comparison of the production biomass (a), glycerol consumption (b) and clavulanic acid (c) between the mutant and wild type strains cultivated in semi-synthetic mediums GSPA, GSPG and GSPO.

of bacteria (Kulkarni and Gadre, 2002). Therefore, CCRD and RSM were used to obtain the best conditions for lipase activity for $S$. clavuligerus wild type and a mutant MMS150. The experimental design proposed was carried out to compare the lipases activities of wild type and mutant strain. The experimental CCRD matrix is presented in Table 1. Eleven experiments were performed for the each lipase, wild type and mutant strain. Treatment 10 showed the highest levels of lipase activity for wild type strain $(\mathrm{pH}$ 7.2 and $25^{\circ} \mathrm{C}$ ). These results suggest that the lipase from $S$. clavuligerus wild type strain has a higher enzyme activity $(2,498.5 \mathrm{U} / \mathrm{mLh})$ at a relatively low temperature and neutral $\mathrm{pH}$. For the mutant MMS150 the optimal conditions of $\mathrm{pH}$ and temperature for extracellular lipase $(2,348.5$ $\mathrm{U} / \mathrm{mLh}$ ) were: $\mathrm{pH} 9.0$ and $35^{\circ} \mathrm{C}$, showing that this enzyme is a basic lipase. Microbial lipases are produced over a wide range of $\mathrm{pH}$ and temperatures. Abramic et al. (1999) also investigated extracellular lipase by Streptomyces rimosus and this lipase showed optimum activity around $\mathrm{pH} 9.5$. Mahadik (2002) assayed the production of acidic lipase by Aspergillus niger under $\mathrm{pH} 5.5$ and $30^{\circ} \mathrm{C}$; Hiol et al. (2000) studied the extracellular lipase produced by a thermophilic Rhizopus oryzae in $\mathrm{pH} 5.6$ and temperature between $28-35^{\circ} \mathrm{C}$. Other works related that lipases can be produced in pH 6.0 and $65^{\circ} \mathrm{C}$ (Becker and Märkl, 2000). Ours results are in accordance.

Analysis of variance (ANOVA) was employed for the determination of significant parameters and to estimate the lipase activity as a function of temperature and $\mathrm{pH}$. Data are shown in Table 2 and 3. The computed $F$-value for wild type lipase (21.95) was 5.05-fold higher than the $F$-value in statistic tables, indicating that the model was significant at high confidence level (95\%), with $\mathrm{R}^{2}=0.9039$, thus $90.4 \%$ of the total variation is explained by the model (Table 3 ). The ratio of $F$ for mutant MMS150 lipase was 12.65, with $\mathrm{R}^{2}=0.9338$ (Table 3 ). Both determination coefficients suggest a satisfactory representation of the process model.

The coded model was used to generate contour diagrams for the analysis of the variable effects on lipase activity, (Figures 6 and 7 for wild type and mutant MMS150, respectively). The experimental results of the CCD desing were fitted with the second-order polynominal Eq. (3) for the lipase from $S$. clavuligerus wt. Only the temperature as linear and interactive terms was not significant. The lipase activity was affected by temperature and $\mathrm{pH}$ as quadratic terms $(\mathrm{p}<0.05)$. In this work these terms were significant, which indicates that they can act as limiting factors and even small variations in their values will alter lipase activity to a considerable extent.

The secondary order polynomial equation is given below:

Table 2 - Analysis of variance (ANOVA) for the model regression by $S$. clavuligerus wild type.

\begin{tabular}{lccccc}
\hline Source & SS & DF & MS & F-value & F-value in statistic table \\
\hline Model & $3,055,090.0$ & 3 & $1,018,363.3$ & 21.95 & 4.35 \\
Residual & $324,792.0$ & 7 & $46,398.6$ & & \\
Total & $3,379,882.0$ & 10 & & & \\
\hline
\end{tabular}

$\mathrm{R}^{2}=0.9039$; SS, Sum of Squares; DF, Degrees of Freedom; MS, Mean Square.

Significance level $=95 \%$. 
Table 3 - Analysis of variance (ANOVA) for the model regression by S. clavuligerus MMS 150.

\begin{tabular}{lccccc}
\hline Source & SS & DF & MS & F-value & F-value in statistic table \\
\hline Model & $2,700,995.00$ & 2 & $1,350,497.50$ & 56.45 & 4.46 \\
Residual & $191,401.00$ & 8 & $23,925.12$ & & \\
Total & $2,892,396.00$ & 10 & & & \\
\hline
\end{tabular}

$\mathrm{R}^{2}=0.9338$; SS, Sum of Squares; DF, Degrees of Freedom; MS, Mean Square.

Significance level $=95 \%$.

$$
\begin{aligned}
& Y=2,192.2+191.36 T-308.12 T^{2}+ \\
& 490.00 p H-312.90 p H^{2}-53.41 p H \times T
\end{aligned}
$$

where, $Y$ represents lipase activity (in $\mathrm{U} / \mathrm{mLh}$ ), $p H$ the hydrogenionic potential, and $T$ is the temperature as coded settings.

To confirm the applicability of the model, lipase activity was determined at $30^{\circ} \mathrm{C}, \mathrm{pH} 7.0$, which are the optimal values suggested by it. In this case, the model prediction showed an enzymatic activity of $1786.6(\mathrm{U} / \mathrm{mLh})$. Experimentally, 2,278.8 (U/mLh) of lipase activity was observed, confirming the closeness of the model to the experimental results.

For the lipase by S. clavuligerus MMS150 the regression equation for the level of lipase production Y:

$$
\begin{aligned}
& Y=1,652.82-265.43 T+38.74 T^{2}+ \\
& 492.66 p H-92.35 p H^{2}+179.55 p H \times T
\end{aligned}
$$

where, $Y$ represents lipase activity (in $\mathrm{U} / \mathrm{mLh}$ ), $p H$ the hydrogenionic potential, and $T$ is the temperature as coded settings.

In this model (Eq. (4)), temperature and $\mathrm{pH}$ as quadratic terms and the interaction as a linear term were not significant, showing that these terms were not factors limiting lipase activity. The applicability of the model was also tested by simulation of the quadratic model. The activity recuperation percentage was $88.25 \% 8$ (data not showed), confirming the empirical validity of the model.

\section{Discussion}

The equivalence in the amplifications band patterns of expected molecular masses (Figure 1) validates that the isolated MMS150 mutant is a S. clavuligerus ATCC 27064 derivative, indicating that both strains are very similar with respect to these markers and should have the same genetic machinery for clavulanic acid production.

When cultivated in semi-synthetic mediums GSPA, GSPG and GSPO the mutant strain has shown greater clavulanic acid production, specially when cultivated in GSPA medium, where the production was about six fold higher than the wild type strain after $60 \mathrm{~h}$ fermentation (Figure 5). The production of clavulanic acid was also higher with the mutant than with the wild type strain (Figure 4), obtaining a $891.3 \mathrm{mg} \mathrm{L}^{-1}$ concentration in $60 \mathrm{~h}$ of fermentation in the modified complex medium, while the wild type strain obtained $820.1 \mathrm{mg} \mathrm{L}^{-1}$ in $72 \mathrm{~h}$. This difference, that may look inexpressive at first, becomes important in view of the clavulanic acid production costs, that in this case might be cheaper due to the economy done in glycerol upon large scale industrial production.

When cultivated in complex medium, the mutant strain presented less lipolytic activity in comparison with the wild type (Figure 3). However, when cultivated in the modified complex medium, mutant strain showed a lipase production about 2.4 times higher than that of the wild type (Figure 4). Such productivity is significant $(\mathrm{p}=0.014)$, indicating that the random mutation also caused the MMS 150 strain a greater lipolytic activity dependent on the composition of the culture medium. Thus, MMS 150 strain was shown to have higher induced lipolytic activity, bacterial growth and clavulanic acid production compared to the wild type when both cultivated in the modified complex medium.

Models for the production of extracellular lipase were developed and the contour plot in Figures 6 and 7 represents the predicted model, indicating the variable levels to an optimal process. The figures also show the shapes contour of $\mathrm{pH}$ against temperature for the two lipase activities. It is clearly seen that there is an effect of conditions optimization for the extracellular lipases. The optimal activity results were: $\mathrm{pH} 7.2$ and $25^{\circ} \mathrm{C}$ for the lipase produced by Streptomyces clavuligerus wild type; and $\mathrm{pH} 9.0$ at $35^{\circ} \mathrm{C}$ for the mutant MMS150.

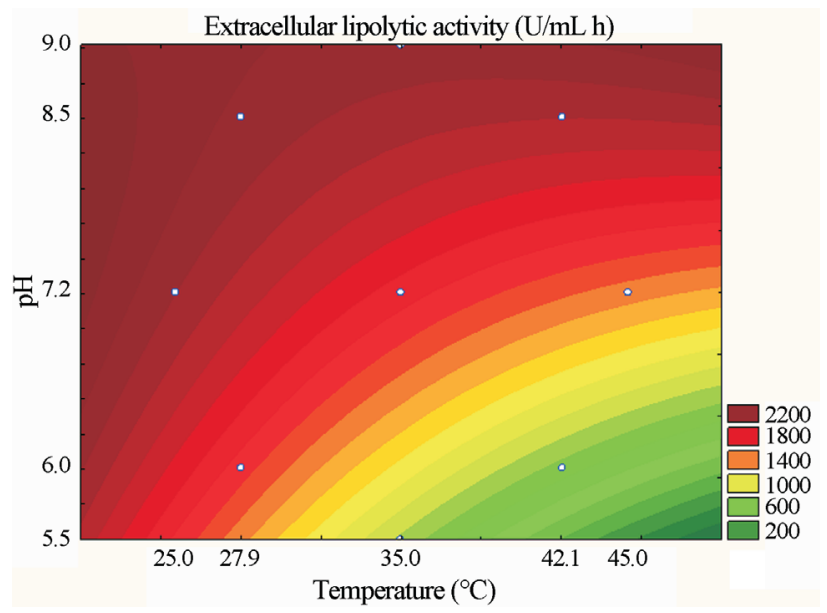

Figure 6 - Contour plot for the effects of temperature and $\mathrm{pH}$ on lipolytic activity of $S$. clavuligerus wild type. 


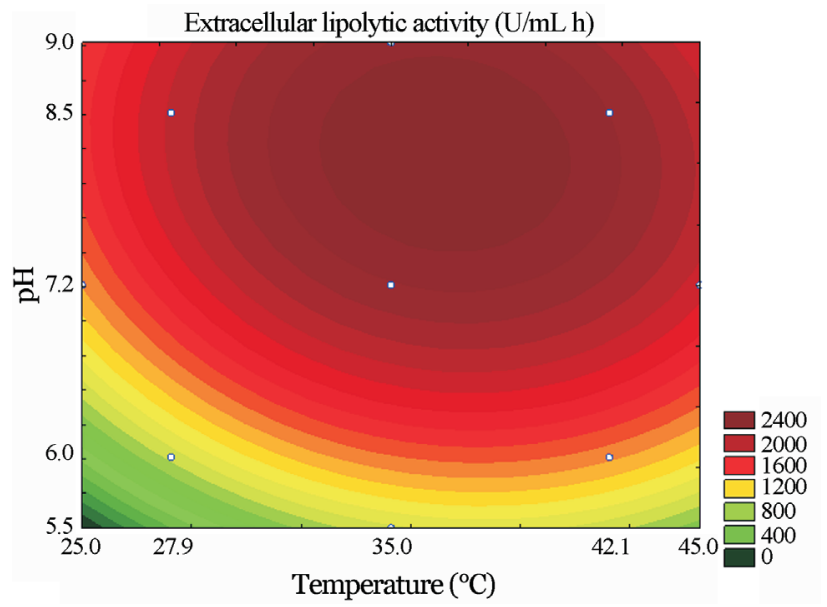

Figure 7 - Contour plot for the effects of temperature and $\mathrm{pH}$ on lipolytic activity of $S$. clavuligerus mutant MMS150.

For the wild strain of $S$. clavuligerus in the complex medium, data of clavulanic acid and lipase showed that the maximum production of lipase and $\mathrm{AC}$ occur between 60 and $72 \mathrm{~h}$ (Figures 3 and 4). The similar results are observed with the strain $S$. clavuligerus MMS 150 . This production standard AC and lipase with oils in fermentations is according to Large et al. (1999), and Peacock et al. (2003). These authors showed that oils added in the fermentations medium increases the titles of antibiotics. This why oils can induce lipase activity in bacteria and fungi and one of product this lipase catalysis is glycerol, because is a precursor of the biosynthesis of clavulanic acid. So, this work confirmed that levels of AC are directly proportional to lipase.

The $S$. clavuligerus strain is very good lipase production in comparison with other microorganisms (Dominguez et al., 2005; Silva et al., 2005). This is a possibility for further studies on the $S$. clavuligerus lipase purification and kinetics for example, aiming broad industrial field interest once lipase is widely used in pharmaceutics, cosmetics and perfumery, oil chemistry, leather, cellulose and paper industry, and even treatment of industrial residues (Hasan et al., 2006).

\section{Acknowledgments}

The authors are thankful to Universidade Federal de São Carlos (UFSCar) and Fundação de Amparo à Pesquisa do Estado de São Paulo (FAPESP) for supporting this work. Leandro Seiji Goto has a fellowship from FAPESP (Proc. 06/59693-1).

\section{References}

Abramic M, Lescic I, Korica T, Vitale L, Saenger W, Pigac J (1999) Purification and properties of extracellular lipase from Streptomyces rimosus. Enzyme Microb Technol 25:522-529.

Aires-Barros MR, Taipa MA, Cabral JMS. Isolation and purification of lipases (1994) In: Woolley P, Petersen SB, editors Li- pases - Their structure, biochemistry and application. Cambridge, UK: Cambridge University Press, pp. 243-70.

Ausubel FM (2002) Short protocols in molecular biology: a compendium of methods from Current protocols in molecular biology, 5th edn. Wiley, New York.

Baggaley KH, Brown AG, Schofield CJ (1997) Chemistry and biosynthesis of clavulanic acid and others clavams. Nat Prod Rep 14:309-333.

Bas D, Boyaci IH (2007) Modeling and optimization I: Usability of response surface methodology. J Food Eng 78:836-845.

Becker P, Märk1 M (2000) Modelling of olive oil degradation and oleic acid inhibition during chemostat and batch cultivation of Bacillus thermoleovorans IHI-91. Biotechnol Bioeng. 70:630-637.

Brock TD, Madigan MT, Martinko JM, Parker J (1994) Biology of microorganisms. 7th ed. London: Prentice Hall.

Bush K (1989) Characterization of $\beta$-lactamases. Antimicrob Agents Chemother 33:259-263.

Chater KF (2001) Regulation of sporulation in Streptomyces coelicolor A3(2): a checkpoint multiplex?. Curr Opin Microbiol 4:667-673.

Dominguez A, Pastrana L, Longo MA, Rúa ML, Sanroman MA (2005) Lipolytic enzyme production by Thermus thermophilus HB27 in a stirred tank bioreactor. Biochem Eng J 26:95-99.

Elander RP (2003) Industrial production of $\beta$-lactam antibiotics. Appl Microbiol Biotechnol 61:385-392.

Foulstone M, Reading C (1982) Assay of amoxicillin and clavulanic acid, components of augumentin, in biological fluids with performance liquid cromatography. Antimicrob Agents Chemother 22:753-762.

Gupta N, Rathi P, Gupta R (2002) Simplified para-nitrophenyl palmitate assay for lipases and esterases. Anal Biochem 311:98-99.

Hasan F, Shah AA, Hameed A (2006) Industrial applications of microbial lipases. Enzyme Microb Technol 39:235-251.

Hiol A, Jonzo MD, Rugani N, Duet D, Sarda L, Comeau LC (2000) Purification and characterization of an extracellular lipase from a thermophilic Rhizopus oryzae strain isolated from palm fruit. Enzyme Microb Technol 26:421-430.

Jaeger K-EH, Ransac S, Dijkstra BW, Colson C, Van Heuvel M, Misset O (1994) Bacterial lipases. FEMS Microbiol Rev 15:29-63.

Jaeger K-E, Liebton K, Zonta A, Schimossek K, Reetz MT (1996) Biotechnological application of Pseudomonas aeruginosa lipase: efficient kinetic resolution of amines and alcohols. Appl Microbiol Biotechnol 46:99-105.

Kieser HM, Bibb KF, Buttner MJ, Chater KF, Hopwood DA (2000) Practical Streptomyces Genetics. Norwich: The John Innes Foundation. 613 p.

Korn-Wendisch F, Kutzner HJ (1992) The Family Streptomycetaceae. In: Starr MP, Stolp H, Truper HG, Balows A, Schlegel HG (eds) The Prokaryotes. Springer-Verlag, Berlin, v. 1, p. 921-995.

Kulkarni N, Gadre RV (2002) Production and properties of an alkaline, thermophilic lipase from Pseudomonas fluorescens NS2W. J Ind Microbiol Biotechnol 28:344-348.

Large KP, Mirjalili N, Osborne M, Peacock LM, Zormpaidis V, Walsh M, Cavanagh ME, Leadlay PF (1999) Lipase activity in Streptomycetes. Enzyme Microb Technol 25:569-575. 
Lee SD, Park SW, Oh KK, Hong SI, Kim SW (2002) Improvement for the production of clavulanic acid by mutant Streptomyces clavuligerus. Lett Appl Microbiol 34:370-375.

Li R, Khaleeli N, Townsend CA (2000) Expansion of the clavulanic acid gene cluster: identification and in vivo functional analysis of three new genes required for biosynthesis of clavulanic acid by Streptomyces clavuligerus. J Bacteriol 182:4087-4095.

Locci R (1989) Streptomyces and related Genera. in Bergeys Manual of Systematic Bacteriology, Williams ST, Sharpe MD, Holt JG (eds) Williams \& Wilkins, Baltimore, USA p. 2451-2492.

Mahadik ND, Puntambekar US, Bastawde KB, Khire JM, Gokhale DV (2002) Production of acidic lipase by Aspergillus niger in solid state fermentation. Process Biochem 38:715-721.

Maranesi GL, Baptista-Neto A, Hokka CO, Badino AC (2005). Utilization of vegetable oil in the production of clavulanic acid by Streptomyces clavuligerus ATCC 27064. World J Microbiol Biotechnol 21:509-514.

Mingrui Y, Shaowei Q, Tianwei T (2007) Purification and characterization of the extracellular lipase Lip2 from Yarrowia lipolytica. Process Biochem 42:384-391.

Pan SC, Bonanno S, Wagman GH (1959) Efficient utilisation of fatty oils as an energy source in penicillin fermentations. Appl Microbiol; 7:176-180.

Paradkar AS, Jensen SE (1995) Functional Analysis of the Gene Encoding the Clavaminate Synthase 2 Isoenzyme Involved in Clavulanic Acid Biosynthesis in Streptomyces clavuligerus. J Bacteriol 177:1307-1314.

Peacock, L, Ward J, Ratledge C, Dickinson FM, Ison A (2003) How Streptomyces lividans uses oils and sugars as mixed substrates. Enzyme and Microbial Technology 32:157-166.

Rolinson, GN (1991). Evolution of $\beta$-lactamases inibitors. Rev Infect Dis 13:5727-5732.

Romero J, Liras P, Martin JF (1986) Utilization of ornithine and arginine as especific precursors of clavulanic acid. Appl Environ Microbiol 52:892-897.

Rosa JC, Neto AB, Hokka CO, Badino AC (2005) Influence of dissolved oxygen and shear conditions on clavulanic acid production by Streptomyces clavuligerus. Bioprocess Biosyst Eng 27:99-104.

Silva WOB, Mitidieri S, Schrank A, Vainstein MH (2005) Production and extraction of an extracellular lipase from the entomopathogenic fungus Metarhizium anisopliae. Process Biochem 40:321-236.

Stonesifer J, Baltz R (1985) Mutagenic DNA repair in Streptomyces. Proc Nat Acad Sci USA 82:1180-1183.

Tahlan K, Park HU, Wong A, Beatty PH, Jensen SE (2004) Two sets of paralogous genes encode the enzymes involved in the early stages of clavulanic acid and clavam metabolite biosynthesis in Streptomyces clavuligerus. Antimicrob Agents Chemother 48:930-939.

Williams ST, Goodfellow M, Alderson G (1989) Genus Streptomyces Waksman and Henrici. In: Williams ST, Sharpe ME, Holt JG (eds) Bergey's Manual of Determinative Bacteriology. Baltimore, Williams and Wilkins, 4:2453-2492.

All the content of the journal, except where otherwise noted, is licensed under a Creative Commons License CC BY-NC. 